\section{A Micrographic Study of Wear of Graphite}

THE sheet-structure of graphite is well known ${ }^{1}$ : upon microscopic examination of crystalline fragments, individual sheets exhibit some properties of a tough membrane when not torn transversely. Individually they may be lifted or peeled to uncover a flat surface, which is highly reflecting, and in thin layers transparent.

These properties make graphite ideally suited for microscopic observations of its wearing characteristics during the sliding process.

The details of this process for graphite had not been apparently discovered. For example, it was not known whether wear accrues from the mechanical action of edges, the adhesion through edge surfaces or across massive facial areas, the tooling action of disoriented crystals, or by other unexpected effects.

Observations have been made here on natural graphite crystals oriented on the stage of a microscope under bright field epi-illumination. Initially the basal plane of the crystal was mounted rigidly and held flat and normal to the incident light beam. With selected adjustments of the illumination, steps, faults and surface impurities became prominent, and slight disturbances to a shallow depth within the crystal were found to produce faint shadows indicating the nature and extent of the stresses or damage.

During this work at magnifications up to 320 , the graphite faces were explored by metal micro-probes or polished needles using the smooth conical section near the tip. The geometry of the arrangement made possible the estimation of the real contact area in relation to the area visibly disturbed by the contact.

This disturbed area was found to be very much greater than that bounded merely by the contact dimensions of the probe. Upon application of pressure of the probe against the graphite, a few sharp shadow lines frequently developed within the graphite surface adjacent to the contact spot. These spread to the sides or in advance of the probe and travelled with it as it traversed the basal plane surface. These shadow lines were usually straight, and extended to distances as much as a hundred times the contact radius. Some were completely elastic, disappearing upon withdrawal of the probe or oscillating back and forth if the probe was made to vibrate gently upon the surface.

By interference microscopy it was found that the shadow lines in representative cases could be the boundaries of basal plane area which had undergone minute localized tilting near the contact region, amounting to a few hundred angstroms of tilt across the entire field of view.

These optical line patterns on the graphite undergoing rubbing are interpreted tentatively as fault or dislocation regions at the intersectional boundaries between original planes and disturbed planes. This interpretation implies reversible atomic separations transversely through the basal planes in patterns sufficiently broad to be observed as narrow lines with an optical microscope. Disturbances of this kind should involve a contribution to the frictional work, and they indicate one of the characteristic features in the sliding process which may precede the onset of wear.

The appearance of these optical line patterns emanating beyond the region of real contact suggests that the shearing and ploughing terms, generally accepted as descriptive of the main processes involved in sliding solids, may not be sufficient to describe the wear and friction occurring on the surfaces of lamellar solids. A new description of the graphitewearing process following from the detailed microscopic observations will appear in due course.

ROBERT H. SAVAGE

General Electric Research Laboratory, Schenectady, New York.

$$
\text { Nov. } 18 .
$$

${ }^{1}$ Lonsdale, K., Endeavour, 6, 139 (1947).

\section{Low-temperature Specific Heats of Body-centred Cubic Chromium-Iron and Chromium-Manganese Alloys}

THE electronic structure of the transition elements in the metallic state is not yet fully understood. It may be hoped that additional information with regard to the electronic specific heat coefficients and other physical properties of these elements, as well as of their alloys, will help in the interpretation of their structure.

On the basis of the band structure of the transition elements and of the usual interpretation of the electronic specific heat coefficient, it should be possible to trace the shape of the partially filled $d$-band, that is, the density of states as a function of the electron concentration, by determining experimentally the electronic specific heat coefficient $\gamma$ for a series of solid solution alloys of a given crystal structure.

In the present work, low-temperature specific heats were determined for body-centred cubic binary solid solutions in the chromium-iron and chromiummanganese systems. Specific heats were measured in the temperature range of $1 \cdot 6^{\circ}-4 \cdot 2^{\circ} \mathrm{K}$. to an estimated accuracy of \pm 2 per cent for most alloys. For a few alloys, where the specific heat depends very sensitively on the alloy composition, the accuracy may have been as low as \pm 5 per cent. The alloy specimens were prepared by induction melting from electrolytically refined metals and homogenized by annealing for at least three days at $1,170^{\circ} \mathrm{C}$. in a purified gas mixture of 92 per cent helium and 8 per cent hydrogen, and quenched to room temperature.

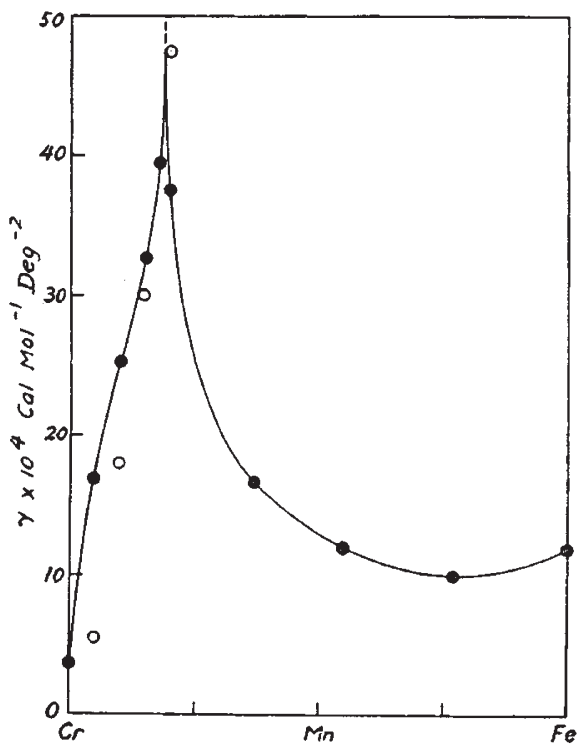

Fig. 1. Coefficient of temperature-linear term of low-temperature specific heat vs. electron concentration. O. Chromium-iron alloys; O, chromium-manganese alloys 